www.jmscr.igmpublication.org

Index Copernicus Value: 79.54

ISSN (e)-2347-176x ISSN (p) 2455-0450

crossref DOI: https://dx.doi.org/10.18535/jmscr/v7i4.90

\title{
A Large Residual Cyst Associated with Mandible: A Case Report and Literature Review
}

\author{
Authors \\ Nithin Kumar*, Nizar Alchaar \\ ISHIK UNIVERSITY, 100 mt. Street, near Filkey Baz (Square), across Qazi Muhammad, 44001, \\ Erbil, KRG/IRAQ \\ *Corresponding Author \\ Nithin Kumar
}

Address: Laxmi nilaya, \#1-96/6, Near anganawadi, guddayangadi, Panjimogeru post, Mangalore, Karnataka - 575013, India

Mob: +919744065322, Email: drnithinkumar1984@gmail.com

\begin{abstract}
Residual cyst is a term most commonly used for any cyst that has been retained within the jaws after the associated tooth has been lost. Most of these cysts develop as radicular cyst. Radicuar cysts are the most common inflammatory cyst occurring in the maxillofacial region. Most residual cysts remain asymptomatic and are discovered on routine radiographic examination. If left untreated they can grow to a considerable size causing bone expansion, erosion and displacement of adjacent teeth. For this reason, it is imperative that this cyst be treated surgically. The recommended treatment option for residual cyst is surgical enucleation. Here we present a case of large residual cyst of mandible with a brief review of literature.

Keywords: Residual cyst, Radicular cyst, Jaw cyst, Periapical cyst, Cyst enucleation.
\end{abstract}

\section{Introduction}

Any cyst that arises as a result of epithelial proliferation within an inflammatory lesion can be termed as an inflammatory jaw cyst. Radicular cysts and residual cyst are the most common inflammatory cysts that can occur in the jaw. ${ }^{[1]}$ Residual cyst is a term used for a radicular cyst that is either left behind in the jaw when it's associated tooth was removed or was formed in residual epithelium of cell rests from a periodontal ligament of the lost tooth. Radicular cysts can occur in the periapical area of any teeth, at any age but are rarely associated with primary dentition. ${ }^{[2]}$ After the associated tooth has been lost, they will continue to grow in the jaw as residual cyst. ${ }^{[3]}$ These cysts can be found at any tooth bearing area of the jaws, with $60 \%$ predilection to maxilla and $40 \%$ in mandible. ${ }^{[1]}$

Most residual cysts remain asymptomatic and are discovered as an incidental finding on routine radiographic examination unless they are secondarily infected. However, as some of them grow, they can cause mobility and displacement of teeth and once infected, lead to pain and swelling, after which the patient usually becomes aware of the problem. ${ }^{[4]}$ Surgical enucleation and primary closure without bone graft is a effective management strategy even in case of large 
residual cyst. ${ }^{[5]}$ A meticulous removal of a cyst is an important aspect of surgical treatment to prevent recurrence. Removal of very large cysts may produce unnatural communication between various anatomic cavities or damage vital structures such as nerves or vessels. ${ }^{[6]}$ Apart from this, a large bony window may be required for removal of the lesion in toto producing a large cortical defect. An innovative method of surgical removal of residual cyst with minimum bony window and avoidance of damage to adjacent vital structure has been introduced here. In this case report, we present a novel method to remove a large residual cyst in mandibular body without any morbidity.

\section{Case Report}

A 54-year-old male patient had come to our hospital for a routine dental check up. Past Medical and drug history was not significant. Past dental history was that of multiple uneventful extractions and fixed partial denture placements. His general physical and extraoral examination did not yield any significant findings. On intraoral examination, it was noted that he had fixed partial dentures on second, third and fourth quadrant of his jaws. On palpation, we were able to notice mild buccal cortical expansion in relation to missing lower first molar tooth region. This swelling was non tender, measuring about $2.5 \mathrm{~cm}$ $x 2.5 \mathrm{~cm}$, spherical in shape, extending from crest of alveolar ridge to just below the depth of the vestibule superoinferiorly and from second premolar to second molar anteroposteriorly. Vitality test performed on the adjacent teeth concurred their vitality. No other significant findings were observed on clinical examination.

On radiological examination, orthopantomogram (figure 1) showed presence of a large round radioleucent lesion measuring about $3 \mathrm{~cm}$ diameter, with a well defined radiopaque border causing mild displacement of adjacent second premolar and second molar teeth. The lesion appeared to have impinged on the mandibular canal, but without causing any sensory disturbance for the patient. Based on the clinical and radiological findings, final diagnosis of residual cyst was arrived at.

Surgical enucleation and primary closure without bone graft was planned as the treatment choice after discussion with the patient. The patient had consented for the procedure. The operative field was isolated, and prepared. Local anaesthesia (2\% lignocaine with 1:80000 adrenaline) was administred for nerve block as well as local infiltration for effective anaesthesia and hemostasis. Using BP blade no. 15, crevicular incision was given from second molar to second premolar with an anterior releasing incision. Following reflection of triangular mucoperiosteal flap, a bony window(figure 2) measuring about $1 \mathrm{~cm}$ diameter was created in the buccal cortex using a small round bur. Care was taken not to remove any bone from the alveolar crest so as to achieve flap closure over an intact bone surface. The cystic lining was gently separated from around the edges of the bony window with the concave surface of the curette facing the crypt wall. Further separation near the bony window was achieved by introducing small sterilized gauze between the cystic lining and the lateral wall of the crypt along with lateral pressure applied on the gauze with the concave surface of the curette facing the bone. After adequate separation of the cystic lining from the accessible area near the bony window, a stab incision was given with a blade to relieve the pressure inside the cyst thereby preventing the unintentional rupture and tearing of the cyst lining on further manipulation. Following this, the cystic lining was held with a mosquito forceps and gently tugged in all direction, which was possible due to the decrease in the intra cystic pressure. As this maneuver was continued, the cyst was completely freed from its bony nest and then was gently pried out through the smaller bony window. Instrumentation with the curette inside the bone cavity especially near the floor was not done to avoid damaging the inferior alveolar nerve. The bone cavity (figure 3) was visually inspected and 


\section{JMSCR Vol||07||Issue||04||Page 525-528||April}

thoroughly irrigated with normal saline. Following adequate hemostasis, the flap was repositioned and sutured with 4-0 interrupted silk sutures. Post operative antibiotics and analgesics were prescribed to the patient and he was recalled after 1 week for suture removal.

The enucleated cystic lesion (figure 4) was stored in $10 \%$ formalin solution and sent for histopathological examination. Histopathological report suggested the lesion as radicular cyst. During the follow up visit for suture removal, the patient was free from all signs and symptoms with adequate tissue healing.

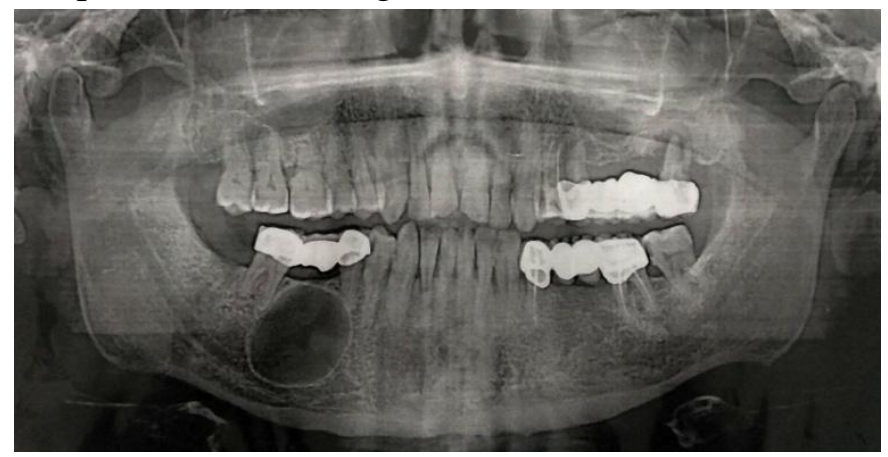

Figure 1: OPG showing the large residual cyst in right mandibular body.

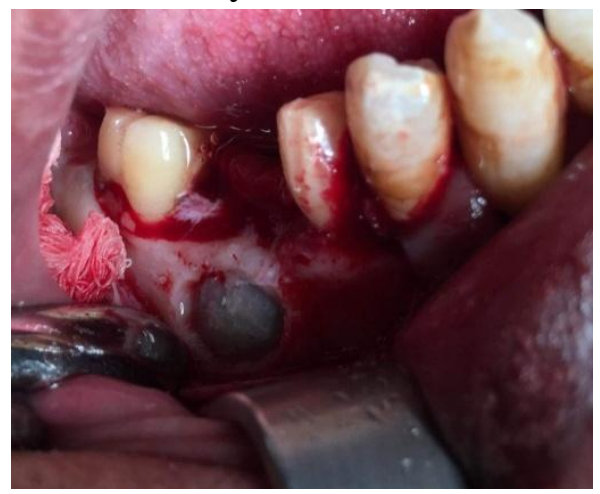

Figure 2: Intra operative view showing bone window created.

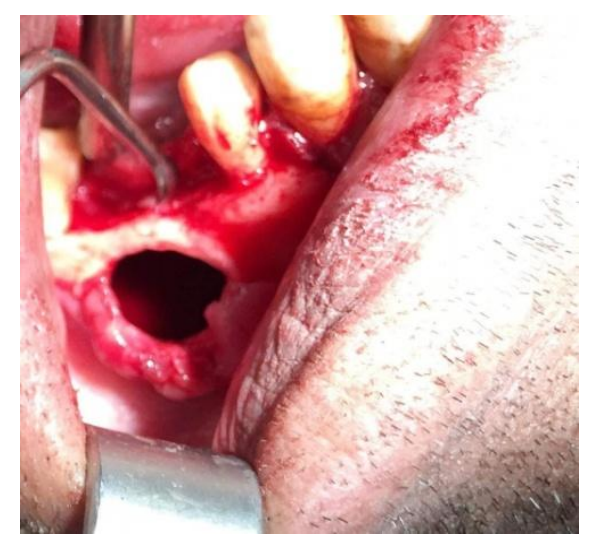

Figure 3: Following complete excision of the lesion.

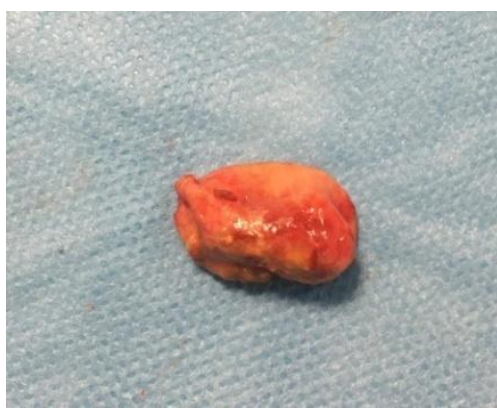

Figure 4: Excised pathological specimen.

\section{Discussion}

Radicular and residual cysts are by far the most common cystic lesions in the jaws with its frequency ranging from $52-68 \%$ of all jaw cysts. Most common site of occurrence of residual cyst was found to be maxillary anterior region and during the third and fourth decade of life. ${ }^{[1,7]}$ In our case, the residual cyst had occurred in the partially edentulous mandibular body region with a missing first permanent molar. The pathogenesis of radicular residual cyst has been described as having three definite phases; phase of Initiation, cyst formation and the enlargement. ${ }^{[8]}$ These true radicular cyst will continue to grow even after the causative tooth has been removed. This "self sustaining" nature of residual cyst makes it imperative that they need to be completely eliminated by surgical enucleation. ${ }^{[3]}$ Types of treatment that can be conducted for the residual cyst is either marsupialisation or enucleation depending on the size of the cyst. ${ }^{[9]}$ But for any residual cyst, enucleation is considered as the "state of the art" treatment even in large cyst of $3 \mathrm{~cm}$ and more in diameter. ${ }^{[5]}$

In the case presented here, the cyst was of relatively large size, measuring approximately $3 \mathrm{~cm}$ diameter. Ideally an endoscopically assisted enucleation would have reduced the morbidity associated with the surgery. Due to the non availability of the equipment, skill set required to operate endoscope and the cost involved, we had to opt for a novel enucleation technique presented herein. ${ }^{[10]}$ Conventional enucleation method involves use of a curette for removal of the cystic lesion from its bony crypt through a adequately large bony window. This bony window is usually 
made through the thinned out bone cortex, which should be of adequate size to facilitate removal of the lesion in toto. This may affect the bone healing during the post operative period. ${ }^{[5]}$ Also as the Curette is a sharp margined instrument, there always remains a risk of injuring vital anatomic structures or puncturing the pathological soft tissue mass, which will make the complete removal of the cystic mass difficult and time consuming. ${ }^{[6]}$ In our case, the cystic lesion was completely intra bony and there was no signs of infection. Bony window was created with minimal bone removal to restrict the opening to approximately $1 \mathrm{~cm}$ diameter, without hindering accessibility. A novel method of enucleation was adopted in this case of large residual cyst to prevent injury to the approximating inferior alveolar nerve. With the concave surface of the curette facing the bone, gentle lateral pressure was applied on a sterilize gauze placed between the cystic lining and the lateral wall of the pathological bone cavity to free the soft tissue from areas near the bony window. Cystic lining was stabbed through the window to relieve the intracystic pressure so as to easily mobilize the cyst inside the bony crypt. Grasping the cystic lining firmly, the lesion can be gently tugged in all direction to free it from the bony crypt and can be easily retrieved through the bone window in toto.

This novel method is more predictable in removal of the soft tissue lesion in toto because of the blunt technique involved, compared to using a sharp curettes inside the cystic cavity with higher chances of rupturing cystic lesion making the removal of the remaining tissue tags difficult and more time consuming. At the same time, the present method prevents risk of damage to approximating vital anatomic structures, resulting in reduced postoperative morbidity to the patient.

\section{Conclusion}

In therapeutic respect, large residual jaw cysts require a complete enucleation. Owing to the benign nature of this lesion, it is imperative that we strive to minimize surgical morbidity as best as possible. In order to achieve this, a novel method has been tried in this case report with an acceptable outcome.

Conflict of interest: None identified.

Source of support: nil

\section{References}

1. Shear M. Cysts of the Oral Regions: Radicular and Residual Cysts. 1992; 3rd ed Boston Wright 136-62.

2. Joshi UK, Patil SK, Siddiqua A. Nasopalatine cyst a rare entity. Int J Dent Clin 2010;2:34-6

3. Nair PN. Review: New perspectives on radicular cysts: Do they heal? Int Endod J. 1998; 31: 155.

4. Dimitroulis G, Curtin J. Massive residual dental cyst: Case report. Aust Dent J 1998; 43(4):234-7.

5. T. Ettl et al. Jaw cysts - Filling or no filling after enucleation? A review. Journal of Cranio-Maxillo-Facial Surgery 40 (2012) 485-493

6. Sweta Tolasaria, Utpal K. Das. Surgical and Nonsurgical Management of Bilateral Periapical Lesions in the Maxillary Anterior Region. J Surg Tech Case Rep. 2011 Jan-Jun; 3(1): 44-48.

7. Jones AV, Craig GT, Franklin CD. Range and demographics of odontogenic cysts diagnosed in a UK population over a 30 -year period. $\mathrm{J}$ Oral Pathol Med 2006; 35: 500-7

8. Jansson L, Ehnevid H, Lindskog S, Blomlöf L. Development of periapical lesions. Swed Dent J 1993; 17: 85-93

9. Adappa, D., Chatra, L., Shenai, P., Veena, K.M., Rao, P.K. and Prabhu, R.V. 2014. Residual Cyst: A Case Report. Int J Adv Health Sci., 1(4): 24-27.

10. Abdelwahab M, Elfattah AMA, Khafagy YW, El-Degwi A. Endoscopic enucleation of large jaw cysts: Promising outcomes. Auris Nasus Larynx. 2018 Jun;45(3):578-584. 